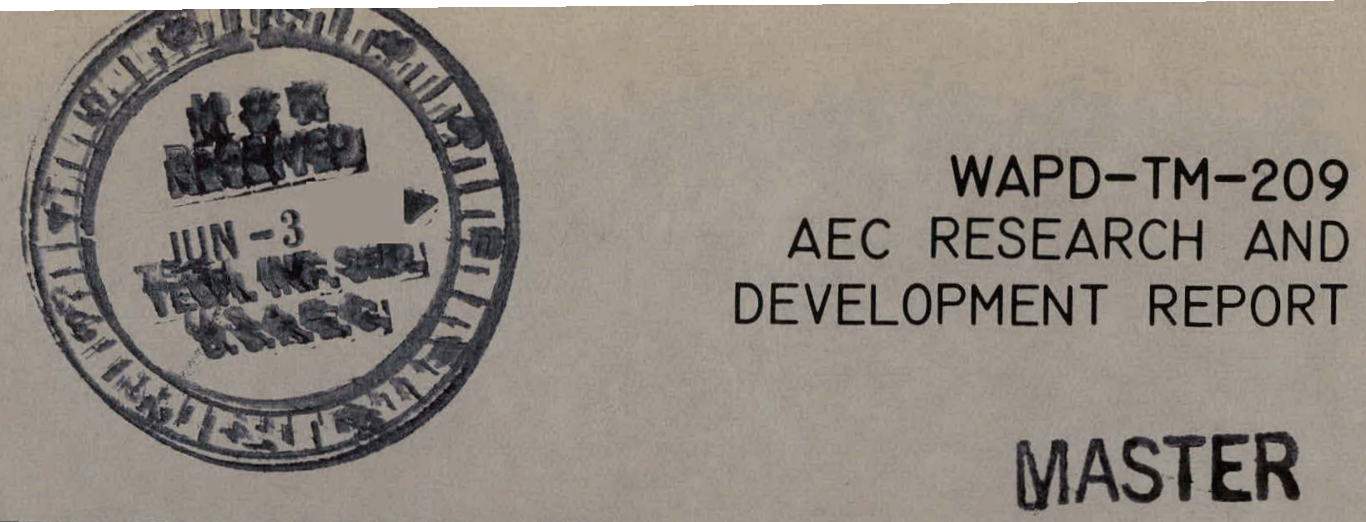

\title{
AN EXPERIMENT TO MEASURE EFFECTIVE DELAYED NEUTRON FRACTIONS
}

February 1960

CONTRACT AT-11-1-GEN-14

BETTIS ATOMIC POWER LABORATORY PITTSBURGH, PENNSYLVANIA

Operated for the U.S. ATOMIC ENERGY COMMISSION bY WESTINGHOUSE ELECTRIC CORPORATION 


\section{DISCLAIMER}

This report was prepared as an account of work sponsored by an agency of the United States Government. Neither the United States Government nor any agency Thereof, nor any of their employees, makes any warranty, express or implied, or assumes any legal liability or responsibility for the accuracy, completeness, or usefulness of any information, apparatus, product, or process disclosed, or represents that its use would not infringe privately owned rights. Reference herein to any specific commercial product, process, or service by trade name, trademark, manufacturer, or otherwise does not necessarily constitute or imply its endorsement, recommendation, or favoring by the United States Government or any agency thereof. The views and opinions of authors expressed herein do not necessarily state or reflect those of the United States Government or any agency thereof. 


\section{DISCLAIMER}

Portions of this document may be illegible in electronic image products. Images are produced from the best available original document. 
WAPD-TM-209

UC-34: Physics and Mathematics

TID-4500 (15th Ed.)

AN EXPERTMENT TO MEASURE EFFECTIVE DELAYED NEUTRON FRACTIONS

S. Kaplan and A. F. Henry

February 1960

Contract AT-11-1-GEN-14

Price $\$ 1.00$

Available from the Office of Technical Services,

Department of Commerce,

Washington 25, $D$. C.

\section{NOTE}

This document is an interim memorandum prepared primarily for internal reference and does not represent a final expression of the opinion of Westinghouse. When this memorandum is distributed externally, it is with the express understanding that Westinghouse makes no representation as to completeness, accuracy, or usability of information contained therein.

BETTIS ATOMIC POWER LABORATORY

PITTSBURGH, PENINSYLVANIA

OPERATED FOR THE U. S. ATOMIC ENERGY COMMISSION BY

WESTINGHOUSE ELECTRIC CORPORATION 
STANDARD EXTERNAL DISTRIBUTION

No. Copies

$\cdots$

UC-34: Physics and Mathematics, TID 4500, 15th Edition

SPECIAI EXTERNAL DISTRIBUTION

Director, Development Division, Pittsburgh Naval Reactors Operations Office, AEC

Totial $\frac{3}{628}$

LEGAL NOTICE

This report was prepared as an account of Government sponsored work。 Neither the United States, nor the Commission, nor any person acting on behalf of these agencies:

A. Makes any warranty or representation, expressed or implied, with respect to the accuracy, completeness, or usefulness of the information contained in this report, or that the use of any Liformation, apparatus, method, or process disclosed in this report may not infringe privately owned rights; or

B. Assumes any liabilities with respect to the use of, or for dam ages resulting f'rom the use $O I^{\circ}$ any information, apparatus, method, or process disclosed in this report.

As used in the above, "person acting on behalf of these agencies" includes any employe or contractor of these agencies to the extent that such employe or contractor prepares, handles or distributes, or provides access to, any information pursuant to his employment or contract with the Commission. 


\section{ABSTRACT}

An experimental measurement of the effective delayed neutron fraction $(\bar{\beta})$ has been made for a clean critical assembly by determining the asymptotic period associated with introduction of a known amount of reactivity. The "known amount" of reactivity was obtained by replacing, uniformly. throughout the reactor, a small quantity of $U-235$ with an alloy of $B^{10}$ and Hf designed to match the absorption properties of U-235. The replacement was thus equivalent to a uniform reduction in $v$, the number of neutrons emitted per fission from the fuel. Such a reduction introduces a reactivity change equal exactly to $\delta v / v_{0}$.

Two analyses of the experiment were made using different high energy cross sections in conjunction with four group, two dimensional diffusion theory. The measured value of $\bar{\beta}$ lay between the results of these computations, the error spread (an average root-means-square error of $\pm 5.2 \%$ ) being too great to permit any conclusion regarding the significance of the comparison. 
AN EXPERIMENT TO MEASURE EFFECTIVE DELAYED IFEUTRON FRACTIONS

S. Kaplan and A. F. Henry

I. INTRODUCTION

It is well known that the effective delayed neutron fractions $\left(\overline{\beta_{i}}\right)$, which appear in the reactor kinetics equations and the inhour formula, differ in magnitude from the actual physical fractions $\left(\beta_{i}\right)$. This difference arises from the fact that different fissionable. isotopes emit different numbers of delayed neutrons and from the fact that all delayed neutrons are' emitted with energies considerably below those of prompt neutrons. . The first circumstance indicates that some sort.of averaging procedure is called for: The second implies that delayed neutrons have a smaller probability of causing fast fission and of leaking out of the core; thus their effect on the chain reaction differs from that of the average prompt neutron.

To the extent that only ratios of the kinetics parameters (reactivity, prompt neutron lifetime, and effective delayed neutron fractions) have any absolute significance, definition of the $\overline{\beta_{i}}$ is arbitrary . This degree of freedom is generally removed by requiring that the reactivity associated with a fractional change in the average number of neutrons, $v$, emitted per fission be exactly $\delta v / v_{0}$. It follows from such a normalization that a uniform pertur bation of $v$ introduces a known amount of reactivity, totally independent of any particular mathematical model used to analyse the system: Insofar as $\overline{\beta_{i}} / \beta_{i}\left(\equiv \gamma_{i}\right)$ is independent of $i$ (i.e. $\gamma_{i}=\gamma \equiv \bar{\beta} / \beta$ ), the effective. fraction, $\bar{\beta}$, can be determined directly from the period associated with the 
perturbation of $v$. In principle, a theoretical model of the reactor is needed! only if the small differences, $\left(\gamma-\gamma_{i}\right)$ for the various delayed groups, $i$, are not entirely negligible.

A direct determination of $\bar{\beta}$ thus can be made by introducing a uniform; perturbation in the number of neutrons emitted per fission. No direct method, of doing this being available, a substitution experiment is required. Accordingly, a non-fissionable material, identical in all other nuclear properties to the fissionable material, is required. Uniform substitution of a . fraction $\delta v / v_{0}$ of the fissionable material with its non-fissionable counterpart will then introduce a reactivity equal exactly to $\infty \delta v / v_{0}^{\circ}$.

This substitution procedure is quite old, having first been applied to the Los Alamos water boiler by Fermi, Feynman, and de Hoffman (Ref, 1)(the, so-called, "boron bubble" experiment). More recently the Plastic Mockup Assembly at KAPL was used for the same purpose (Ref. 2), boron again being the element substituted for U-235. The experiment to be described in the present paper differs from these previous ones primarily in the fact that some attempt was made to account for resonance absorption in uranium by using a mixture of boron and hafnium to replace U-235. In addition, a fairly thorough theoretical analysis has been carried out to determine the size of corrections needed to bridge the gap between the ideal and the actual experiment.

In practice, the substitution is made in several stages, and the effects are added. This procedure leads to problems in reproducibility. (A shift of $2.0 \times 10^{-4}$ in reactivity can result in an error of $\sim 10 \%$ in $\gamma$ ) As. a result the final error estimate for the experiment is $\approx 5 \%$ in $\gamma_{0}$ : It is believed that this could be reduced by repeated experimental normalization 
and the use of procedures requiring special dispensation from standard operam ting safety regulations such as the restriction that water be dumped from the reactor tank. if any fuel or poison strip is to be changed. The present report thus represents more a careful analysis of the theoretical and experimental difficulties in determining $\gamma$, rather than an account of the best determination reasonably possible。

II. THE THEORY

\section{A. Formal Considerations}

1. Definitions

The effective delayed neutron fraction, $\overline{\beta_{i}}$, for the $i$-th delayed precursor group can be defined as (Ref. 3),

$$
\overline{\beta_{i}} \equiv \frac{1}{F} \int_{V} \int_{u} \int_{u^{\prime}} \frac{v}{v_{0}} \sum_{j} v^{j}\left(u^{\prime}\right) f_{i}(u) \beta_{i}^{j} \Sigma_{f}^{j}\left(\underline{r}, u^{\prime}, t\right) \varphi_{0}^{\underline{\mu}}(\underline{r}, u) \varphi\left(\underline{r}, u^{\prime}, t, t\right) d v d u d u^{\prime}
$$

with

$$
\begin{array}{r}
F \equiv \int_{v} \int_{u} \int_{u^{\prime}} \sum_{j} \sum_{i}\left[f^{j}(u)\left(I-\beta^{j}\right) \not f_{j}(u) \beta_{i}^{j}\right] \\
v^{j}\left(u^{\prime}\right) \Sigma_{f}^{j}\left(\underline{r}, u^{\prime}, t\right) \varphi_{0}^{k}(\underline{r}, u) \varphi\left(\underline{r}, u^{\prime}, t\right) d v d u d u^{\prime}
\end{array}
$$

In these definitions, integrals are over all volume and lethargies $u$ and $u^{\prime}$. The sums are over all precursor groups:(subscript, i) and all fissionable isotopes (superscript, $j$ ). The $f^{\prime} s$ are spectra, normalized to unity, $f^{j}(u$ ) being proportional to the average number of prompt neutrons per unit lethargy emitted from a fission of isotope, $j$, and $f_{j}(u)$ (frequently taken as a delta 
function in lethargy) being the spectra of delayed neutrons from the $i-t h$ precursor group. ( $f_{i}$ is assumed to be independent of $j_{\cdot}$ ) The adjoint flux, $\varphi_{0}^{A}(r, u)$, refers to some arbitrary stationary state with respect, to which all perturbations are measured. It is the integral over all directions, $\Omega$, of the directional worth $\psi_{0}^{\mathbb{k}}(\underline{r}, \underline{\rho}, u)$ of neutrons at position $\underline{r}$, having lethargy u. Similarly, $\varphi(\underline{r}, u, t)$ is the time dependent directional flux $\psi(\underline{r}, \underline{\Omega}, u, t)$ integrated over all $\underline{\Omega}$ and normalized so that the integral.

$$
\iint_{u} \frac{\varphi_{0}^{\sharp}(r, u) \varphi(r, u, t)}{v(u)} d u d v
$$

(where $v$ is the neutron speed at lethargy, $u$ ) is constant in time.

In terms of this same formalism, the reactivity and prompt neutron lifetime are defined by

$\rho \equiv \frac{1}{\mathrm{~F}} \int_{\mathrm{V}} \int_{\mathrm{u}}\left[-\varphi_{\mathrm{O}}^{\mathrm{A}}(\underline{\mathrm{r}}, \mathrm{u}) \int_{\Omega} \underline{\Omega} \cdot \nabla \psi(\underline{\mathrm{r}}, \underline{\Omega}, \mathrm{u}, \mathrm{t}) \mathrm{d} \Omega-\varphi(\underline{r}, \mathrm{u}, \mathrm{t}) \int_{\Omega} \underline{\Omega} \cdot \nabla \psi_{0}^{\mathrm{A}}(\underline{\mathrm{r}}, \underline{\Omega}, \mathrm{u}) \mathrm{d} \Omega\right.$

$$
\begin{aligned}
& -\delta \Sigma(\underline{r}, u, t) \varphi_{0}^{A}(\underline{r}, u) \varphi(\underline{r}, u, t) \phi \int_{u^{\prime}} \delta\left\{\Sigma_{s}^{0}\left(\underline{r}, u^{\prime}, u, t\right)+\frac{v}{v_{0}} \sum_{, j} \sum_{i}\left\langle f^{j}(u)\left(1-\beta^{j}\right)<f_{i}(u) \beta_{i}^{j}\right\rangle\right. \\
& \left.\left.x v^{j}\left(u^{\prime}\right) z_{f^{i}}^{i}\left(\underline{r}^{\prime}, u^{\prime}, t\right)\right\} p_{0}^{k}(\underline{r}, u) e p\left(\underline{r}, u^{\prime}, t\right) d u^{\prime}\right] d y d u \\
& \Lambda=\frac{1}{\vec{F}} \int_{V} \int_{u} \frac{\varphi_{0}^{\mu}(\underline{r}, \bar{u}) \phi(\underline{r}, u, t)}{v(u)} \text {. }
\end{aligned}
$$

where changes in the total cross section, $\Sigma(\underline{r}, u, t)$; the $P_{0}$ component of the scattering cross section from lethargy $u^{\prime}$ to $u, \Sigma_{S}^{O}\left(\underline{r}, u^{\prime}, u, t\right)$, and the fission 
cross section, $\Sigma_{f}^{j}(\underline{r}, u, t)$, are measured relative to values for the stationary system for which $\psi_{0}^{k}(\underline{r}, \underline{\Omega}, u)$ is the adjoint.

2. Computation of $\overline{\beta_{i}}$

Equation (1) shows that the $\overline{\beta_{i}}$ can be time dependent. In practice, this time dependence is generally quite negligible. Accordingly, the quantity desired is given by Eq. (l) with $\varphi(\underline{r}, \underline{u}, t)=\varphi_{0}(\underline{r}, u)$ and $\Sigma_{f}^{j}(\underline{r}, u, t)=\Sigma_{f}^{j}(\underline{r}, u, o)$. The standard calculation is based on noting that if one of the $\beta_{i}^{j}(i=k)$ is doubled and $v / v_{0}$ is adjusted to $v^{\prime} / v_{0}$ so that the net reactivity change vanishes, Eq. (3) gives (with the flux resulting from the double perturbation approximated by $\left.\varphi_{0}(\underline{r}, u)\right)$

$0=\int_{V} \int_{u} \sum_{\mathfrak{u}^{\prime}} \sum_{j}\left[\frac{v^{\prime}}{v_{0}}<f^{j}(u)\left(I-\beta^{j}\right)+f_{i}(u)\left(I+\delta_{i k}\right) \beta_{i}^{j}\right\rangle$

$$
\left.-\frac{v}{v_{0}}\left\langle f^{j}(u)\left(1-\beta^{j}\right) \phi f_{i}(u) \beta_{i}^{j}\right\rangle\right] \times v^{j}\left(u^{\prime}\right) \Sigma_{f}^{j}(\underline{r}, u, o) \varphi_{0}^{A}(\underline{r}, u) \varphi_{0}(\underline{r}, u) d v d u d u^{\prime} \text {. }
$$

Equations (1) and (2) show that Eq. (5) may be written

$$
0=\frac{v^{\prime}}{v_{0}} F+\frac{v^{\prime}}{v} \overline{\beta_{k}} F \cdot-\frac{v}{v_{0}} F
$$

or

$$
\overline{\beta_{k}}=\frac{\lambda^{\prime}-\lambda}{\lambda^{2}} \quad \text { where } \quad \lambda^{\prime} \equiv \frac{v^{\prime}}{v_{0}} ; \quad \lambda \equiv \frac{v}{v_{0}} .
$$

Thus $\overline{\beta_{k}}$ can be computed from the eigenvalues of two stationary problems, one of them the initial criticality problem; the other having its spectrum augmented by an amount, $\int_{u} \sum_{j} f_{k}(u) \beta_{k}^{j} d u$. 
3. Insertion of a known Amount of Reactivity

A perturbation, $\frac{v}{v_{0}} \frac{\delta v^{j}(u)}{v^{j}(u)}$, made equal to $\delta v / v_{0}$ for all isotopes, 1 $j$, at all lethargies is equivalent to perturbing the eigenvalue by an amount $\delta v / v_{0}$. Equation (Al) in the Appendix together with $\mathrm{Eq}$. (2) in the text shows that the value of reactivity associated with this perturbation is exactly $\delta v / v_{0}$, regardless of the time behavior of $\psi(\underline{r}, \underline{\Omega}, \underline{u}, t)$. Furthermore, it is also shown in the Appendix that Eq. (3) predicts the same reactivity change provided $\delta v / v_{0}$ is small. Thus a small known amount of reactivity can be introduced into a system by perturbing slightly the numbers of neutrons produced in fissions.

\section{B. Practical Considerations}

1. The Ideal Experiment

For a source-free reactor on a stationary period $\left(T, \dot{c}_{i} \sim e^{\omega t}\right)$ Eq. (A3) (and the associated delayed neutron equations) reluce to the inhour formula

$\rho=\omega \Lambda \leftarrow \sum_{i} \frac{\omega \overline{\beta_{i}}}{\omega t \lambda_{i}} \equiv \omega \Lambda \leftarrow \bar{\beta} \sum_{i} \frac{\gamma_{i}}{\gamma} \frac{\omega \beta_{i} / \beta}{\omega r \lambda_{i}} ; \gamma_{i} \equiv \frac{\overline{\beta_{i}}}{\bar{\beta}_{i}} ; \gamma \equiv \frac{\bar{\beta}}{\beta}$

where the $\beta_{i}$ are the actual delayed fractions for the principal fissioning material. Thus if a known amount of reactivity, $\delta \mathrm{v} / \mathrm{v}_{\mathrm{o}}$ leads to a measured period, $1 / \omega$,

$$
\bar{\beta}=\frac{\frac{\delta v}{v_{0}}-\omega \Lambda}{\sum_{i} \frac{\gamma_{i}}{\gamma} \frac{\omega \beta_{i} / \beta}{\omega / \lambda_{i}}}
$$


The energy spectra, $f_{i}(u)$, of the delayed neutron groups are sufficiently similar that, if only one fissionable material is present $\gamma_{i}=\gamma$ for all $i$. In addition, in most practical experiments, $\omega \Lambda \ll \delta v / v_{0}{ }^{\circ}$ Under these circumstances, Eq. (14) becomes

$$
\bar{\beta} \simeq\left[\frac{\delta v}{v_{0}}\right] /\left[\sum_{i} \frac{\omega \beta_{i} / \beta}{\omega p \lambda_{i}}\right] .
$$

Thus the determination of $\bar{\beta}$ is independent of any mathematical computations. If the approximations on which Eq. (10) depends are not justified, some theoretical computations must be made to determine $\Lambda$ and the $\gamma_{i} / \gamma_{\text {. The }}$ These have, however, the nature of correction factors and need not be determined with great precision.

Determination of $\bar{\beta}$ thus depends essentially on perturbing $v$. This cannot be done directly; however, Eq. (3) shows that perturbing the fission cross section can be made exactly equivalent. This latter perturbation is accomplished by replacing uniformly some of the fissionable material with material, identical in absorbing and scattering properties, but non-fissionable. A practical experiment differs from this ideal in two main respects. First, the replacement material will possess only an approximate equivalence to the absorbing and scattering properties of the fissionable material. Second, a completely uniform replacement in one step is too expensive to warrant the effort. Possible errors arising from these two sources must be examined.

The presence of the perturbed fission cross section in the denominators, $F$, of $\rho$ and $\bar{\beta}$ leads to a slight difference in $\rho$ and $\bar{\beta}$ individually. Thus, if $v / v_{0}$ is perturbed, $\boldsymbol{\rho}=\left(v_{2}-v_{1}\right) / v_{0}$ and $\overline{\bar{\beta}}={ }^{\prime}\left(v_{2} / v_{1}\right) \overline{\beta_{0}}$ where $\overline{\beta_{0}}$ is for the unperturbed state; if $\Sigma_{f}$ is perturbed uniformly, $\rho=\left(v_{1} / v_{0}\right)\left(\Delta \Sigma_{f} / \Sigma_{f_{2}}\right)$; $\bar{\beta}=\overline{\beta_{0}}$. Nevertheless the ratio $\rho / \bar{\beta}$ (which is the actual quantity measured) is the same for both cases provided $\Sigma_{f_{2}} / \Sigma_{f_{1}}=v_{2} / v_{1}=\lambda_{1} / \lambda_{2}$. 


\section{The Replacement Material.}

Since safety considerations limit the reactivity change-associated with any experiment to $\sim 0.3 \%$, the amount of $\mathrm{U}^{25}$ replaced is also $\sim 0.3 \%$ In the experiment run, about 1 atom in 160 was $U^{25}$. Thus $\sim 1.9 \times 10^{-5}$ of the atoms are replaced by others during an experiment. With such a small quantity of material involved, reactivity effects due to differences in scattering cross sections are negligible. As a consequence the replacement material was specified solely on the basis of its absorption properties. Specifically, appropriate number densities in a mixture of $\mathrm{B}^{10}$ and $\mathrm{Hf}$ were determined by requiring that the macroscopic thermal absorption cross section averaged over the thermal spectrum in the core (Ref. 4)(0 to $0.625 \mathrm{ev}$ ) and the macroscopic epithermal absorption cross section averaged over the epithermal spectrum (Ref. 5) $\left(0.625\right.$ to $5.53 \times 10^{3} \mathrm{ev)} \mathrm{equal} \mathrm{the} \mathrm{corresponding} \mathrm{quantities} \mathrm{for} U^{25}$. The procedure was to determine the microscopic absorption cross sections of $\mathrm{B}^{10}$ and Hf averaged over the core spectrum and from these, the required number densities. The resultant alloy has been named "Delerium" (De)-Delerium-II, to be precise, $\mathrm{De}$ being a mixture of $\mathrm{B}^{\mathrm{I}}$ and $\mathrm{Ag}$, less attractive from a fabrication point of view.

Both $\mathrm{De}^{\mathrm{II}}$ and the fuel it displaced were alloyed with zirconium, and the actual experiment involved exchanging strips of the two alloys. Rigid tolerances on zirconium content were not invoked, and the manufactured delerium-zirconium strips occupied $\sim 1 \%$ more volume than the corresponding fuel alloy strips. Under the assumption-expected to overestimate the effect-othat the extra zirconium is equivalent to a void, it was estimated (from measured void coefficients) that only $\sim 0.05 \%$ of the total reactivity change in any given measurement was ascribable to this zirconium mismatch. 8 
It is clear from the procedure used to determining the composition of delerium that the desired makeup of the material depends to some extent on the composition of the core in which it is to be used. This effect was examined quantitatively by several computations in which the delerium, specified for one core material was used in somewhat different surroundings. The resultant eigenvalues (one for $10 \%$ of the $U^{25}$ replaced by $\mathrm{De}$, the other for $v / y$, reduced 10\%) were indistinguishable within the limits of error imposed on the convergence of the test problems $\left(v / v_{0} \equiv 1 / \lambda\right.$ to $\left.\pm 5 \times 10^{-5}\right)$. It appears, then, not to be essential that a given delerium alloy be used in exactly that composition for which it was designed.

An estimate of the error which can be tolerated in the composition of the alloy can be made by noting that removal of $10 \%$ of the fuel from a core: such as the one used in this experiment would reduce reactivity $\sim 3 \%$. $(\delta v / v$ would be $10 \%$ but the absorption of the fuel would be removed along with its fissioning.) Thus addition of the De yields about $7 \%$ of the total $10 \%$ effect, and about $70 \%$ of any error in the delerium composition will show up in the inferred value of $\delta v / v$ 。

3. Theoretical Corrections Due to Nonuniform and Additive Nature of Replacement Procedure

The ideal of a uniform exchange of $\sim 0.3 \%$ of the fuel with delerium would be extremely difficult and expensive to achieve. In practice two approximations were made. First, the replacement was carried out by exchanging one out of eight fuel strips in a one inch square box of fuel plates (see Fig。 1). Second, only a few boxes were altered at a time, the result. for the entire core being obtained by sumation. The approximations were examined separately, justification for additivity being estimated first and : 
corrections for the specific location of the replaced strips being made on an overall core basis. This procedure permits corrections to be made from a single calculation.

There are two aspects to the additivity problem. The first is the purely theoretical question of whether reactivities obtained from perturbing isolated regions of a core can be added to give the correct overall effect. The second involves the experimental difficulty of reproducibility。 This seçond aspect will be discussed in section IV.

Equation (3) shows that the sum of reactivity effects from local perturbations in $v / v_{0}$ (performed at different times) will equal exactly the single reactivity change for all perturbations performed at once provided the shape function, $\psi(\underline{r}, \underline{\Omega}, u, t)$, associated with the local changes is the same as that for the overall change。 Since perturbations in $v / v_{0}$ effect only the local source of fission neutrons. (which tend to diffuse more than any other neutrons in the core), accompanying changes in Illux shape are expected wo be small. To verify this expectation, two numerical examples were constructed. The first involved a simple water-reflected slab, six inches thick, the fuel

Because of the presence of the perturbed fission cross section in the
denominator, F, this statement is only approximate if $\Sigma_{f}$, rather than
$v / v_{O}$, is perturbed. However, if flux shape changes are neglected, the fractional difference between the sums of ratios $\frac{\rho_{\ell}}{\bar{\beta}_{l}}\left(=\sum_{i} \frac{\left(\beta_{j} / \beta\right) \omega_{l}}{\omega_{l} / \lambda_{l}}\right)$. obtained from local perturbations of $\Sigma_{f}$ and the single ratio $\rho / \beta$ obtained from the overall perturbation $\Delta \Sigma_{f} / \Sigma_{f_{l}}$ is $\sim \sum_{\ell=1}^{N} \frac{\rho_{l}^{2}}{\rho}$ where $\rho_{l}$ is the 1.reactivity associated with one of the $\mathrm{N}$ individual perturbations, For $P_{\ell}=0.002, N=15$, and $\boldsymbol{P}=0.03$, this amounts to an error of $\sim 0.2 \%$ in the inferred value of $\delta v / v_{0}$. 
zone being a mixture: of $\mathrm{Zr}_{3}: \mathrm{H}_{2} \mathrm{O}$ (volume ratio $\sim 1.2: 1$ ) and highly enriched uranium. The reactivities resulting from decreasing $\Sigma_{\text {fiss }} 10 \%$ in six local regions were calculated from the differences in eigenvalues, $\lambda,\left(\equiv v / v_{0}\right)$ of the perturbed and unperturbed cases. (The WANDA code (Ref. 6) in two energy groups was used to obtain solutions.) Since the six regions encompassed the whole core volume, summing the eigenvalue changes, $\left(-\frac{\ldots \delta \lambda_{i} \cdots}{\lambda_{\text {unperturbed }}}\right)$, should yield exactly $=0.10000\left(-\frac{\Delta \text { fiss }}{\Sigma_{\text {fiss }}}\right)$, provided the addition procedure is valid. Results for this example are shown on Fig. I. They suggest that the procedure is valid to within $0.25 \%$ of the total reactivity change. Results from a second numerical example are shown on Fig. 2. In this case a more complicated geometry was studied (a symmetrical slab arrangement composed of two different fuel materials, separated by a hafnium sheet, the central fuel region being bisected by a two inch water gap)。. Again, the individual contributions to the reactivity, although quite sensitive to location, seem to within $0.74 \%$ of the correct total.

Since the subregions perturbed in the experiment.were smaller than those in the examples, the addition procedure should be even more valid there and is thus expected to lead to negligible error in $\gamma$.

In the ideal experiment the delerium is introduced uniformly whereas in actual fact it is localized in an alloy strip replacing one of the eight fuiel strips in a one inch square bundle. Since the experiment was performed on a clean rectangular parallelepiped core, flux shapes were relatively smooth except at the core reflector interface. Thus the correction for the localization of the delerium was small. It was, however, not negligible and had to be accounted for. This was done by obtaining, with the PDQ code $\left(\operatorname{Ref}_{*} 7\right)$, four-group, two-dimensional diffusion theory fluxes and adjoint 


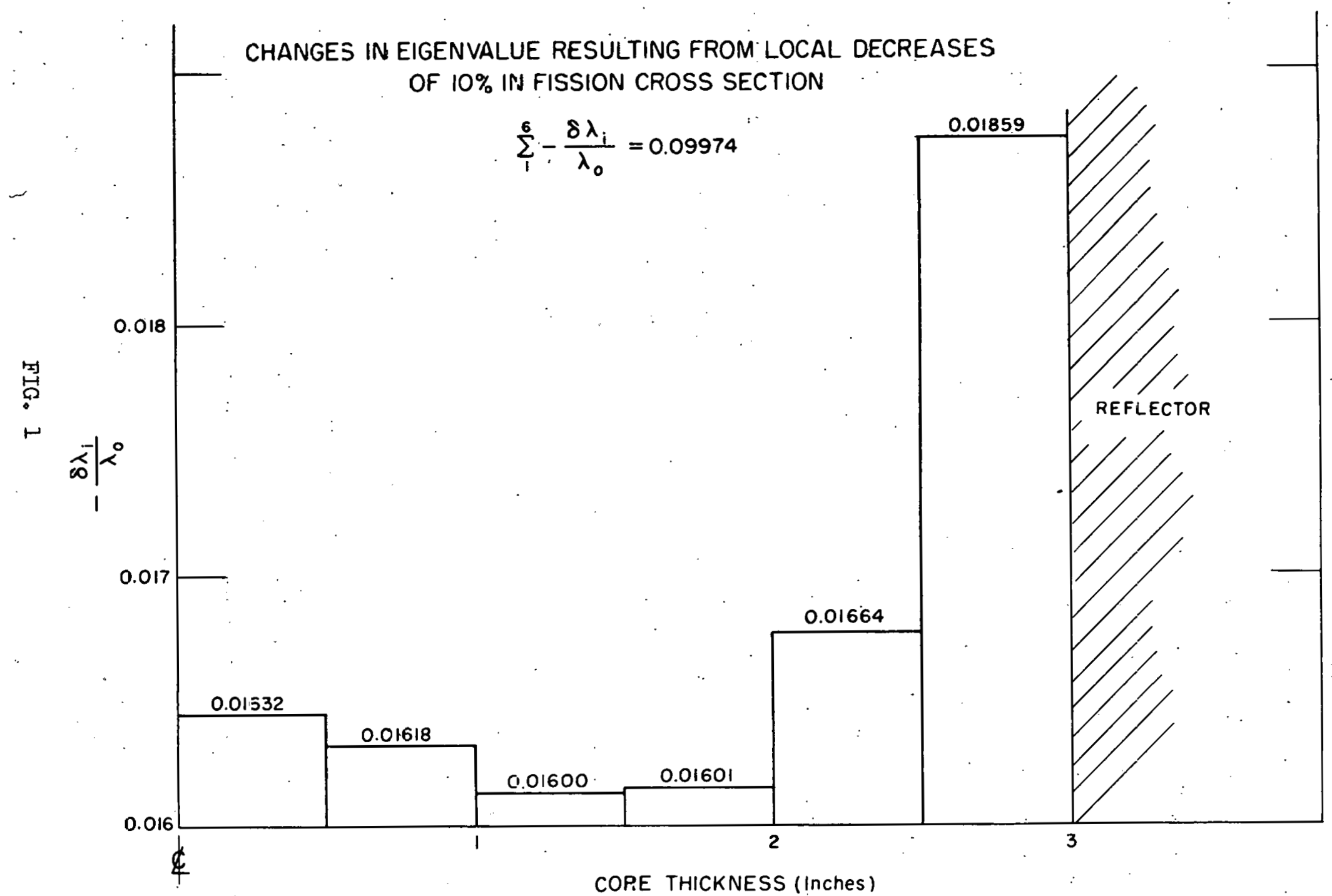



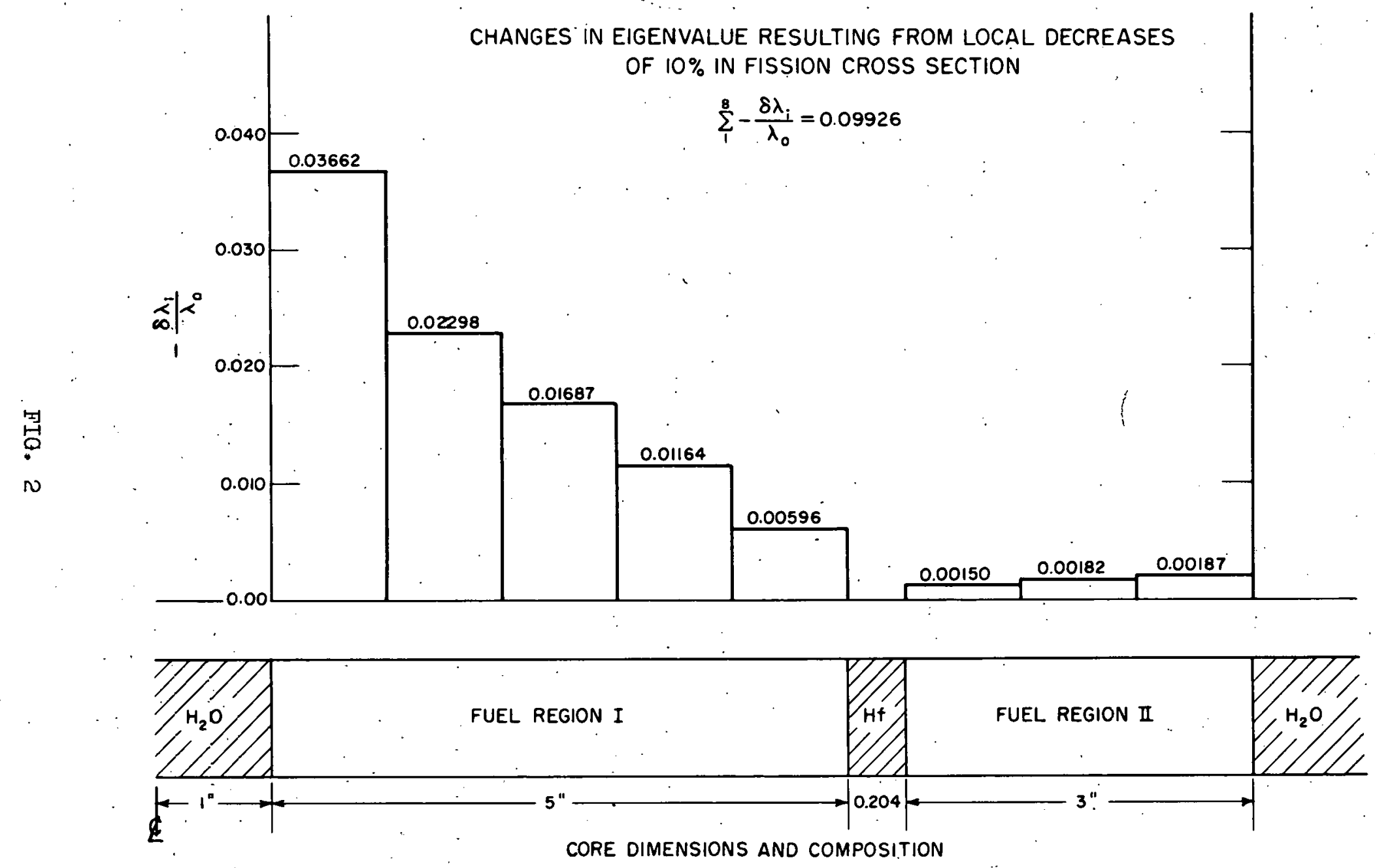
fluxes for a horizontal cross section of the core. (The flux shape vertically was assumed to be a cosine.). Weighting functions were then obtained for each one inch square fuel bundle and along the one inch line, in that fuel bundle, where the delerium was located. The theoretical delerium worths were then adjusted by multiplying by the ratio of the heterogeneous to the homogeneous weiighting function.

The samc fluxes and adjoints were used to correct for differences... between the "as-manut'actured" urul Lleal delerium. Insofar as the correctiong were small, ( 6\% for the heterogeneity and $\sim 5 \%$, for the manufacturing mismatch) the use of an approximate mathematical model is expected to be legitimate.

III. DESCRIPTION OF THE EXPERTMENT

The experiment was performed in the Submarine Project critical facility on the metal slab reactor deslgnated a.s Core No. 36 in the records of that facility. A diagram of this cure is shown in Fig. 3. The core is composed of zirconium modules one of which is shown in detail in Fif. 4. Each unit cell contains eight 36 inch fuel bearing shims. of these, the one replaced during the substitution experiment was alwaye one of the two centermost shims.

The procedure of the experiment was to measure the experimental period of the seactor with no delarium shims present (the "clean" condition). This was done with the control rods at two different positions, fully out and inserted two inches. The reactor was then shut down and the vessel drained. Delerium shims were then substituted for fuel in one or more modules, the vessel refilled and the reactor period measured again at the 14 
SKETCH OF REACTOR CORE AND VESSEL

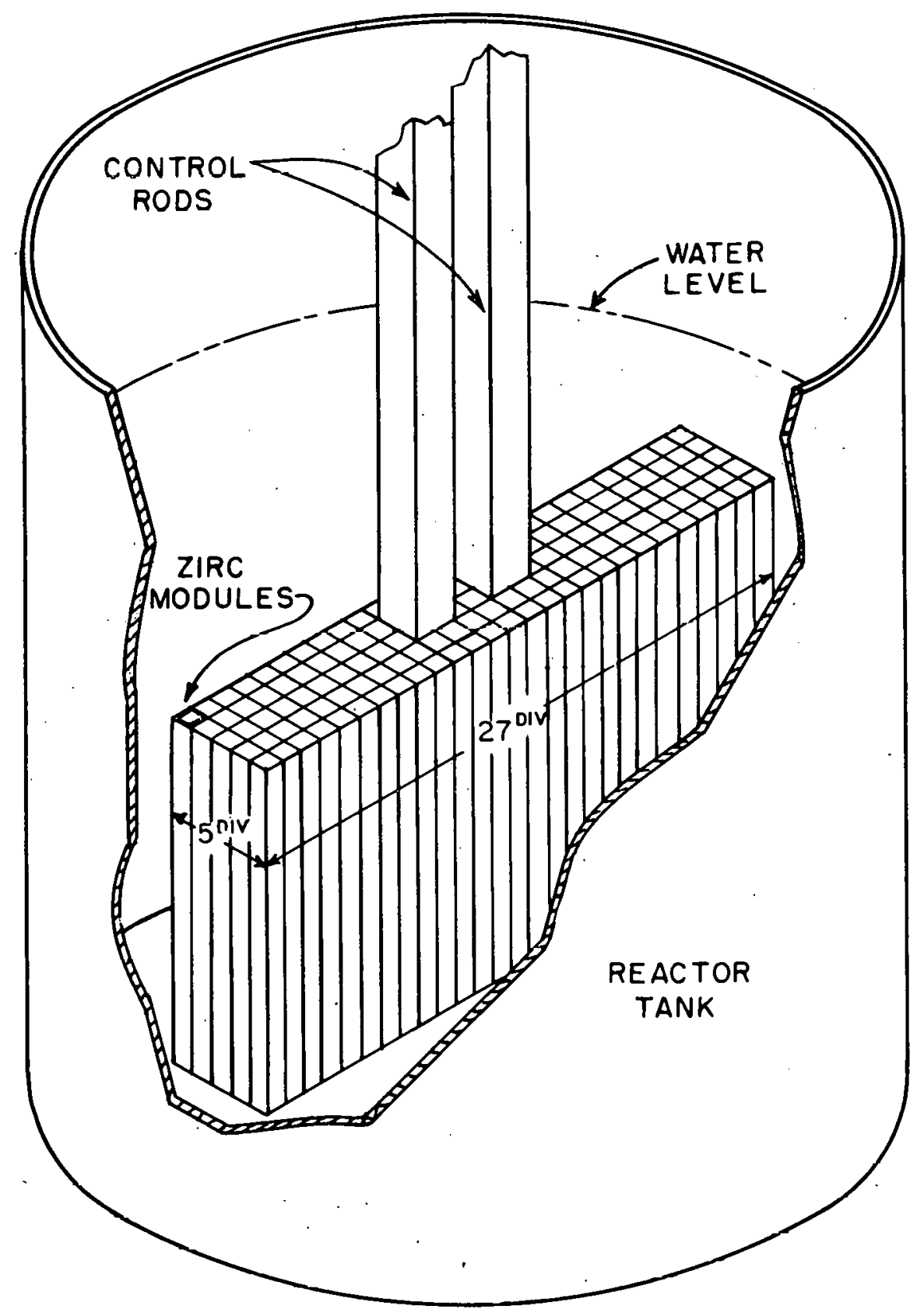

FIG. 3 


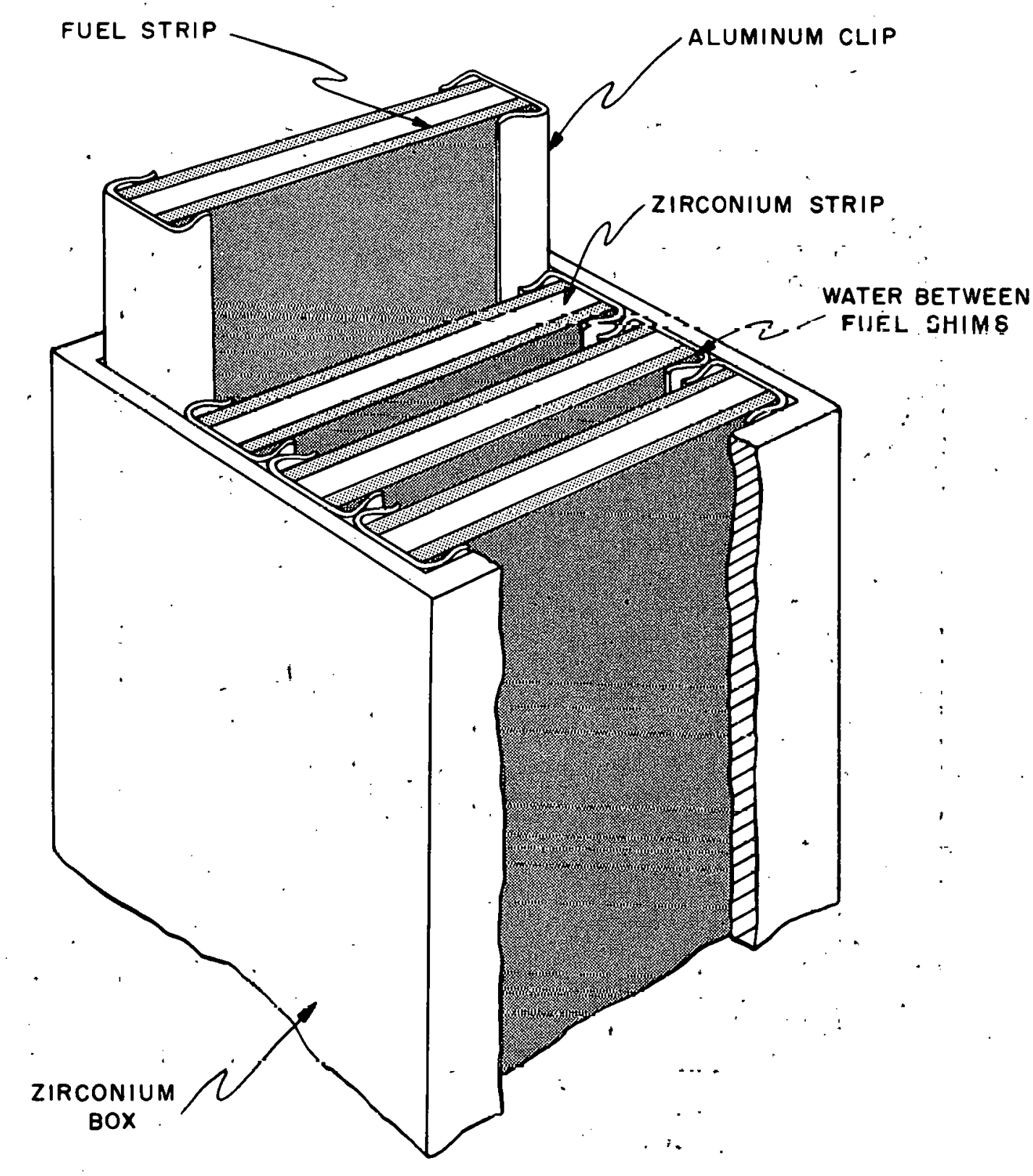

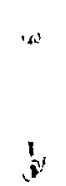


same two rod positions. The difference in reactivity between the clean and the delerium containing core at the corresponding rod position is the reactivity worth of the substitution. For the next experiment the fuel was reinserted and the delerium moved to other modules. The average of the worths obtained from the two rod positions for each substitution are shown in Fig. 5. (The worths obtained from the two rod positions for the most part were within $1 \%$ of each other.)

Figure 5 shows a quarter of the reactor in plan view. The boundaries of the zirconium modules are indicated by dashed lines. Heavy lines indicate the sets of modules which contained delerium simultaneously, i.e. each set constituted a single substitution experiment. These sets were designed, based on theoretical estimates of local worth, so as to give coverage of the core in the minimum number of substitutions: (since each substitution required approximately two hours of facility time) and so as to give the largest reactivity differences possible without getting into the range where negative periods are insensitive to reactivity.

For each set of modules Fig. 5 lists the experimental worth determined from the measured periods through the inhour formula with a $\beta=0.00642$. Also listed is the theoretical worth of each substitution based on a pertur-.. bation theory calculation using four group PDQ fluxes and adjoints. The theoretical values are corrected for the localized position of the delerium within the module and for the mismatch of the delerium absorption and the fuel shim absorption (a 5\% effect). The ratio of the theoretical to

The mismatch results from the fact that the delerium composition was originally designied based on the "older" boron microscopic cross sections (MUFT-SOFOCATE element No. 29) whereas the theoretical values in Fig. 5 are based on more recent microscopic cross sections (MUFT-SOFOCATE element No. 35). 
PLAN VIEW OF CORE QUADRANT

THEORETICAL AND EXPERIMENTAL WOFTHS

OF DELEREIUM SUBSTITUTION AT VARIOUS POSITIONS

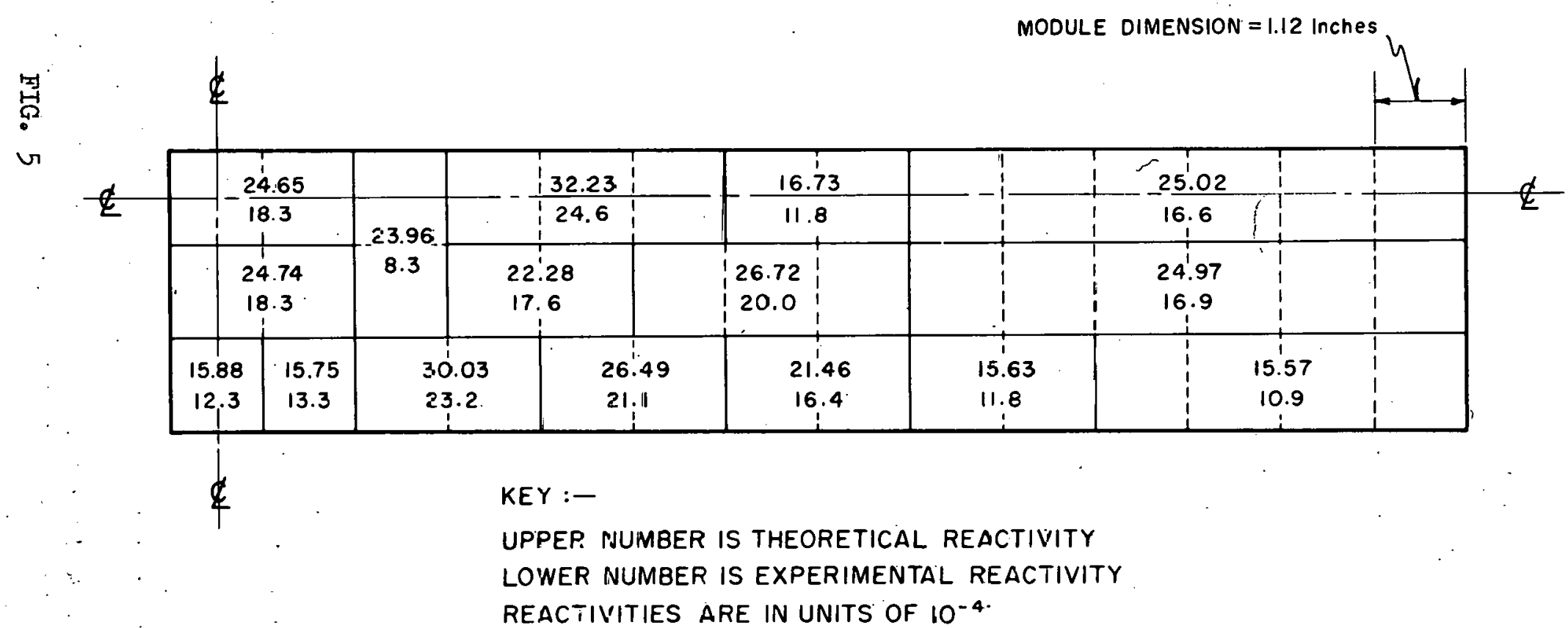


experimental worth for each substitution is a determination of $\bar{\beta} / \beta$. These values however depend directly on the ability of the theory to calculate the local worth. The best value of $\bar{\beta} / \beta$ is obtained by summing the theoretical worths and summing the experimental worths,over all the modules; for which substitutions were made and then taking the ratio of these sums. The value obtained in this way is dependent upon the theory only to make :corrections for the absorption mismatch, the localization of delerium within the module and the fact that the modules for which substitutions were made overlap the lines of symmetry of the core by a thickness of $1 / 2$ module.

The value obtained from the ratio of sums is $\bar{\beta} / \beta=1.33$. A discussion of possible errors in this value is given in the next section.

\section{DISCUSSION OF ERRORS}

We divide the uncertainties into two categories, (a) those which are purely experimental in origin and (b) those. which arise from the theoretical corrections to the experimental data. on the experimental side the main problem is that of reproducibility. It is suspected that during the acts of emptying the reactor vessel, manually rattling the core as during the substitution of delerium for fuel, and then refilling the vessel, small amounts of reactivity are introduced by mechanisms not precisely'understood. In order to define precisely the degree of reproducibility it would be necessary to make an extensive series of repeated measurements: A certain amount, of repeated measurements were actually made during this experiment, however because of the cost.in facility time the number of repetitions was. not large enough to define precisely. the" amount: of reproducibility. On the basis of the measurements that were made and the uncertainty remaining 
it is felt necessary to ascribe to the experimental reproducibility a probable error of $4 \%$ in the final value of $\bar{\beta}$. Another source of experimental error lies in the chemical and spectrographic determination of the composition of the delerium shims. It is estimated that this source leads to a in $3 \%$ uncertainty in the final value of $\bar{\beta} / \beta$.

On the theoretical side corrections have been made for absorption mismatch, for localization of the delerium within the modules and for the fact that the central modules overlap the lines of core symmetry. Assuming that the microscopic cross sections of fuel, boron, and hafnium are known exactly then the mismatch correction suffers only trom the lluitationo of four exnm theory. Since the mismatch correction is about 5\%, the assumption that four group theory weighting functions are valid to $10 \%$ gives an error of $0.5 \%$ due to this source in the final value of $\bar{\beta}$. To this must be added the uncertainty in the microscopic cross sections themselves, estimated as giving $a 0.5 \%$ uncertainty in $\bar{\beta}$. The currection for localization amounted to $6 \%$ and it is estimated that this leads to an uncertainty of $0.6 \%$ in $\bar{\beta} / \beta$.

Finally a $20 \%$ theoretlcal correction is made for the overlapping. This correction may be in error to the extent that four group diffusion theory is unable to predict the spatial shape of the delerium worth function. However for most of the region over which it is made diffusion theory should be quite adequate. If, therefore, it is assumed that the theory gives a. $5 \%$ error in the worth of the overlapping modules relative to the worth of the rest of the core, the result is a $1 \%$ error in the final value of $\bar{\beta}$. Summing all these errors leads to an estimated probable error of $5.2 \%$. 


\section{COMPARISON WITH THEORY}

A general description of a method for computing $\bar{\beta}$ was discussed in Section II. Design practice at Bettis is to use this method in conjunction with a variant of a few group diffusion scheme in which separate fast groups are used to describe the behavior of neutrons emitted from delayed precursors. Group parameters for such neutrons are determined using a delayed neutron spectrum in MUFT (Ref。 5), and a "parallel" group theory, justified by the linear nature of the equations, is constructed. In four groups, the resulting equations are

$$
\begin{array}{ll}
\nabla \cdot D_{1} \nabla \varphi_{1}^{p}-\left(\Sigma_{a_{1}}+\Sigma_{r_{1}}\right) \varphi_{1}^{p}+\frac{1}{\lambda} x_{1} s=0 & \nabla \cdot D_{1}^{d} \nabla \varphi_{1}^{d}-\left(\Sigma_{a_{1}}^{d}+\Sigma_{r_{1}}^{d}\right) \varphi_{1}^{d}+\frac{1}{\lambda} x_{1}^{d} s=0 \\
\nabla \cdot D_{2} \nabla \varphi_{2}^{p}-\left(\Sigma_{a_{2}}+\Sigma_{\dot{r}_{2}}\right) \varphi_{2}^{p}+\Sigma_{r_{1}} \varphi_{1}^{p}+\frac{1}{\lambda} x_{2} s=0 & \nabla \cdot D_{2}^{d} \nabla \varphi_{2}^{d}-\left(\Sigma_{a_{2}}^{d}+\Sigma_{r_{2}}^{d}\right) \varphi_{2}^{d} f \Sigma_{r_{1}}^{d} \varphi_{1}^{d}+\frac{1}{\lambda} x_{2}^{d} s=0 \\
\nabla \cdot D_{3} \nabla \varphi_{3}^{p}-\left(\Sigma_{a_{3}}+\Sigma_{r_{3}}\right) \varphi_{3}^{p}+\Sigma_{r_{2}} \phi_{2}^{p}=0 & \nabla \cdot D_{3}^{d} \nabla \varphi_{3}^{d}-\left(\Sigma_{a_{3}}^{d}+\Sigma_{r_{3}}^{d}\right) \varphi_{3}^{d} f \Sigma_{r_{2}}^{d} \varphi_{2}^{d}=0 .
\end{array}
$$

$$
\begin{gathered}
\nabla \cdot D_{4} \nabla \varphi_{4}-\Sigma_{a_{4}} \varphi_{4}+\Sigma_{r_{3}} \varphi_{3}^{p}+\Sigma_{r_{3}}^{d} \varphi_{3}^{d}=0 \\
s \equiv \sum_{i}\left(v \Sigma_{f_{i}} \varphi_{i}^{p}+v \Sigma_{f_{i}}^{d} \varphi_{i}^{d}\right)
\end{gathered}
$$

In these equations the $D^{\prime} s, \Sigma_{a}{ }^{\prime} s, \Sigma_{r}$ 's and $X^{\prime} s$. represent diffusion constants, absorption cross sections, removal cross sections, and fractions of the total source appearing in the upper two groups. The thermal flux, $\varphi_{4}$, may be split into two components, $\varphi_{4}^{p}$ and $\varphi_{4}^{d}$, defined by the equations 


$$
\left.\begin{array}{c}
\nabla \cdot D_{4} \nabla \varphi_{4}^{p}-\Sigma_{a_{4}} \varphi_{4}^{p}+\Sigma_{r_{3}} \varphi_{3}^{p}=0 \\
\cdots \\
\nabla \cdot D_{4} \nabla \varphi_{4}^{d}-\Sigma_{a_{4}} \varphi_{4}^{d}+\Sigma_{r_{3}}^{d} \varphi_{3}^{d}=0
\end{array}\right\}
$$

If this is done, the elght equations (Eq. (II) less the thermal equation plus Eq. (12)) may be solved by a standard eight group diffusion theory code. The eigenvalues for two cases, the first with $\chi_{1}^{d}=\chi_{2}^{d}=0$, the second with $x_{1}^{d}$ \& $x_{2}^{d}=\beta$, can be used in conjunction with Eq. (7) to give $\bar{\beta}_{\text {. }}$

This procedure was used to compute a value of $\bar{\beta}$ to compare with experiment. The eight group equations were solved in one dimension on an IBM 650 using the LIL ABNER code (Ref, 8). Measured transverse bucklings were used for the two larger dimensions of the core. These were assumed to be the same for both problems. Two sets of calculations were performed, the first with the standard Bettis production deck of nuclear data and the second with a revised deck which accounts for improvements in the nuclear data developed since the first deck was constructed. Pertinenl, constantc appear in Table $I$. The transverse buckling. tor all problemis In all grouph was taken as $0.001867 \mathrm{~cm}^{-2}$. Table II shows the resulting eigenvalues and computed values of $\bar{\beta} / \rho_{\text {. }}$

Althuubih thero ic some encouragement in the fact that the experimental value lies between the two theoretical values, the error is too large to permit any meaningful conclusions regarding the validily of the theory. Th obtain experimental accuracy sufficiently great to distinguish between the two theoretical results appears to be an extremely difficult project. 
Table I

Group Constants Used in Calculation of $\bar{\beta}$

Core Material (Using Production Library)

\begin{tabular}{|c|c|c|c|c|c|c|c|}
\hline $\begin{array}{l}\text { Group } \\
\text { Designation }\end{array}$ & 1 & 2 & 3 & 4 &.$\dot{\mathrm{d}}$ & $\therefore 2^{d}$ & $3^{\mathrm{d}}$ \\
\hline$D(\mathrm{~cm}) \cdots$ & 2.376 & 0.92512 & 0.6969 & 0.3908 & 0.9311 & 0.9012 & 0.6963. \\
\hline$\Sigma_{r}\left(\mathrm{~cm}^{-1}\right)$ & 0.044195 & 0.1118 & 0.05437 & 0 & 0.1157 & 0.1118 & 0.05436 \\
\hline$\Sigma_{a}\left(\mathrm{~cm}^{-1}\right)$ & 0.000914 & 0.001252 & 0.01486 & 0.1520 & 0.000632 & 0.00125 & 0.01486 \\
\hline$v \Sigma_{f}\left(\mathrm{~cm}^{-1}\right)$ & 0.001409 & 0.002312 & 0.02377 & 0.2974 & 0.001323 & 0.002309 & 0.02376 \\
\hline$x^{3}$ & 0.95231 & 0.04751 & 0 & 0 & $\begin{array}{c}0.00642 \\
(\text { or } 0)\end{array}$ & 0 & 0 \\
\hline
\end{tabular}

Reflector Material (Using Production Library)

$\begin{array}{llllllll}D(\mathrm{~cm}) & 1.990 & 0.9193 & 0.5885 & 0.1529 & 0.7390 & 0.8191 & 0.5837\end{array}$

$\begin{array}{llllllll}\Sigma_{\mathrm{r}}\left(\mathrm{cm}^{-1}\right) & 0.09454 & 0.2706 & 0.1518 & 0 & 0.2685 & 0.2703 & 0.1518\end{array}$

$\begin{array}{llllllll}\Sigma_{\mathrm{a}}\left(\mathrm{cm}^{-1}\right) & 0.001013 & 0.000019 & 0.000953 & 0.01968 & 0.000005 & 0.000019 & 0.000952\end{array}$ Core Material (Using Revised Library).

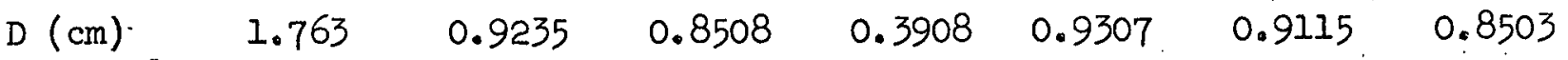

$\begin{array}{llllllll}\Sigma_{\mathrm{r}}\left(\mathrm{cm}^{-1}\right) & 0.04889 & 0.1107 & 0.05363 & 0 & 0.1167 & 0.1102 & 0.05362\end{array}$

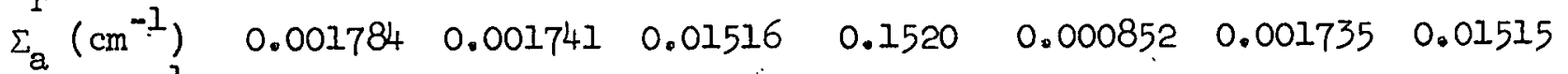

$\begin{array}{llllllll}v \Sigma_{f} \cdot\left(\mathrm{cm}^{-1}\right) & 0.001193 & 0.002019 & 0.02105 & 0.2974 & 0.001089 & 0.002012 & 0.02105\end{array}$

$\begin{array}{llllll}X & 0.95231 & 0.04751 & 0 & 0 & 0.00642\end{array}$

Reflector Material (Using Revised Library)

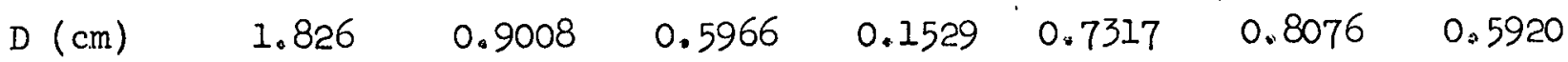

$\begin{array}{llllllll}\Sigma_{r}\left(\mathrm{~cm}^{-1}\right) & 0.09578 & 0.2686 & 0.1518 & 0 & 0.2743 & 0.2655 & 0.1516\end{array}$

$\begin{array}{llllllllll}\Sigma_{\mathrm{a}}\left(\mathrm{cm}^{-1}\right) & 0.001008 & 0.000019 & 0.000953 & 0.01968 & 0.000005 & 0.000018 & 0.000952\end{array}$ 
Table II

Results of Eigenvalue Computation of $(\bar{\beta} / \beta)$

\begin{tabular}{llccc}
$\begin{array}{l}\text { Cross Section } \\
\text { Library }\end{array}$ & $\lambda\left(X_{\mathrm{I}}^{\mathrm{d}}=0\right)$ & $\lambda\left(X_{\mathrm{I}}^{\mathrm{d}}=0.00642\right)$ & $\bar{\beta} / \beta$ & $\begin{array}{l}\text { Experimental } \\
\text { value of } \bar{\beta} / \beta\end{array}$ \\
\hline Production & 0.977818 & 0.986103 & 1.350 & $1.33 \pm 5 \%$ \\
Revised & 0.993444 & 1.001447 & 1.263 & $1.33 \pm 5 \%$ \\
\hline
\end{tabular}

24 
The Transport Theory Form for Parameters in the Kinetics Equations

Equations (1) to (4) represent what might be called the diffusion theory form for the parameters of the kinetics equation. This terminology is not meant to imply any approximations in the expressions but rather that they have been obtained by integrating the neutron and adjoint equation over. all directions before cross multiplication and subtraction. (Ref 43 ), a procedure which yields results reducing in a straightforward manner to the common diffusion theory expressions (Ref.9). If instead the equally valid procedure of integrating over $\underline{\Omega}$ after cross multiplication and subtraction (Ref. 10) is used, expressions for $P$ and $\Lambda$ become

$$
\begin{aligned}
& \ddot{P}_{t} \equiv \frac{i^{\prime}}{F_{t}} \int_{V} \int_{u} \int_{\Omega}\left[-\delta \Sigma(\underline{r}, u, t) \psi_{0}^{\prime}(\underline{r}, \underline{\Omega}, u) \psi(\underline{r}, \underline{\Omega}, u, t) \cdot r \int_{u^{\prime}} \int_{\Omega^{\prime}} \delta\left\{\Sigma_{s}\left(\underline{r}, \underline{\Omega^{\prime}}+\underline{\Omega}, u^{\prime}, u, t\right)\right.\right. \\
& \downarrow \frac{v}{v_{0}} \sum_{j} \sum_{i}\left\langle f^{j}(u)\left(1-\beta^{j}\right) \not f_{i}(u) \beta_{i}^{j}>v^{j}\left(u^{\prime}\right) \Sigma_{f}^{j}\left(r, u^{\prime}, t\right)\right\} \\
& \left.\psi_{0}^{\mathrm{k}}(\underline{r}, \underline{\Omega}, u) \psi(\underline{r}, \underline{\Omega}, u, t) d u^{\prime} d \Omega^{\prime}\right] \text { dvaud } \Omega \\
& \Lambda_{t}=\frac{1}{F_{t}} \int_{v} \int_{u} \int_{\Omega} \frac{\psi_{0}^{A}(\underline{r}, \underline{\Omega}, u) \psi(\underline{r}, \underline{\Omega}, u, t)}{v(u)} \operatorname{dvaud} \Omega
\end{aligned}
$$

where $\Sigma_{s}\left(\underline{r}, \underline{\Omega}^{\prime} \cdot \underline{\Omega} u^{\prime}, u, t\right)$ is the scattering cross section from direction $\underline{\Omega}$ ' to $\underline{\Omega}$ and from lethargy $u^{\prime}$ to $u_{\text {. }}$ If (as has been assumed) prompt and delayed neutrons are emitted isotropically, $\bar{\beta}$ is still given by Eq. (I). 
The normalization of $\psi(\underline{r}, \underline{\Omega}, u, t)$ is now to require $\Lambda_{t} F_{t}$ (rather than $\Lambda F$ ) to' be time independent. The form of the kinetics equations and the inhour formula is the same for both sets of definitions. That is in the diffusion theory ) form

$$
\dot{T}=\frac{\rho-\bar{B}}{\Lambda} T+\sum_{i} c_{i} \lambda_{i} \not Q
$$

where

$$
\begin{aligned}
& c_{i} \equiv \frac{l}{\Lambda F} \int_{V} \int_{u} C_{i}(\underline{r}, t) f_{i}(u) \phi_{0}^{\sharp}(\underline{r}, u) d v d u \quad, \quad \cdots \\
& Q \equiv \frac{1}{\Lambda^{F}} \cdot \int_{V} \int_{u} \varphi_{0}^{\mathbb{A}_{0}}(\underline{x}, u) s(\underline{r}, \underline{\Omega}, u, t) \operatorname{dvdud} \Omega \ldots .
\end{aligned}
$$

$C_{i}$ being the actual delayed neutron precursor concentration and $s$ the external source. In this equation $T(=T(t))$ is the amplitude function, $T(t) \psi(\underline{r} \underline{\Omega}, u, t)$ being the detailed time dependent transport flux.

The second method of deriving the kinetics equations yields

$$
\dot{T}_{t}=\frac{\rho_{t}-\bar{\beta}}{\Lambda_{t}} T_{t}+\sum_{i} c_{i_{t}} \lambda_{1}+Q_{t}
$$

where

$$
\begin{aligned}
& s_{i_{t}} \equiv \frac{1}{\Lambda_{t} w_{t}^{\prime}} \int_{V} \int_{u} \int_{\Omega} c_{i}(\underline{r}, t) f_{i}(u) \psi_{0}^{\mu}(r, \underline{\Omega}, u) \operatorname{dvdud} \Omega \\
& a_{t} \equiv \frac{1}{\Lambda_{t} F_{t}} \int_{v} \int_{u} \int_{\Omega} \psi_{0}^{A}(\underline{r}, \underline{\Omega}, u) s(\underline{r}, \underline{\Omega}, u, t) \text { dvdud } \Omega
\end{aligned}
$$

\footnotetext{
$F_{t}$ may differ from $F$ because of the initial normalization (i.e it may be inconvenient to require that $\left.\Lambda_{\mathrm{t}} \mathrm{F}_{\mathrm{t}}=\Lambda \mathrm{F}\right)$. Note that $\bar{\beta}, . \rho, \Lambda$ are independent of the normalization of $\psi_{0}^{\mathrm{K}}$ and $\psi$.
} 
Being the same physical quantities, $T \psi_{1}=T_{t} \psi_{t}$. Similarly, since $\varphi_{0}^{*}(\underline{r}, u)=\int \psi_{0}^{A}(\underline{r}, \underline{\Omega}, u) d \Omega$, and $\frac{\psi}{F}=\frac{\psi_{t}}{F_{t}}, \quad \Lambda \psi c_{i}=\Lambda_{t} \psi_{t} c_{i_{t}}$, a relationship independent of the normalization of $\psi$ or $\psi_{0^{*}}^{k}$ Multiplying Eq。 (10) by $\Lambda_{t} \psi_{t}$, and subtracting from the product of $\mathrm{Eq} .(8)$ and $\Lambda \psi$ yields

$$
\rho=\rho_{t} t\left(\Lambda-\Lambda_{t}\right) \frac{\dot{T}}{T}+\Lambda_{t}\left(\frac{\psi_{t}}{\psi_{t}}-\frac{\dot{\psi}}{\psi}\right) t \frac{\Lambda_{t} \psi_{t} Q_{t}-\Lambda \psi Q}{T \psi}
$$

For an isotropic source, the last term in Eq. (A7) vanishes. . It follows that, since $\Lambda$ is a small quantity (and $\Lambda-\Lambda_{\mathrm{t}}$ at least three times smaller), $\rho$ will very nearly equal $P_{t}$ except during a fast transient or in: the presence of a large anisotropic source. Thus, in the formalism of Eqs. (3), (4), (A3), and (A4), to a high order of approximation, a perturbation in the eigenvalue, $v / v_{0}$, introduces an absolute amount of reactivity, $\delta v / v_{0}$, provided the reciprocal period, $\omega$, associated with this perturbation is small.

From the foregoing it may appear that the transport theory formalism (Eqs. (Al) to $(\mathrm{A} 4)$ ) is the more suitable. From a formal point of view, this is indeed the case. However in most practical cases, diffusion theory must be used to describe the reactor. The formalism of $\mathrm{Eq}$. . (4) is then more convenient, since, for a $P-I$, Selengut-Goertzel approximation, the first two terms reduce to (Ref. 9 )

$$
\frac{1}{\bar{F}} \int_{V} \int_{u}-\delta D(u, \underline{r}) \nabla \varphi_{0}^{A}(\underline{r} ; u): \nabla \varphi(\underline{r}, u ; t) d V d u
$$

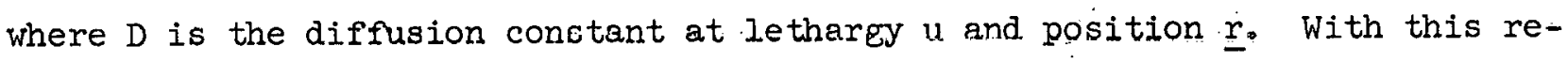
duction, the expression for $\rho$ involves only P-O components of the directional flux and adjoint. Use of Eq. (Al) in a $\mathrm{P}-\mathrm{I}$ approximation would require computation of the P-1 components. 


\section{ACKNOWLEDGMENT .}

The authors wish to express their gratitude to Mr. R. L. Eichinger for the production of the special alloy strips used in the experiment and to the radiochemistry and mass spectrometer groups for their careful isotopic analysis of the completed strips. We wish to thank the members of the Submarine Project Experimental Physics Group under Dr. R. To Jones and the Operational Services Group under Mr. R. M. Matyas for their interest and cooperation in actually carrying out the experiment and for several key suggestions in connection with the detailed experimental procedure. We are indebted to Mr. T. M. Milier of the Surface Ship Critical Facility Group for his contribution in processing the raw experimental data on the Librascope computer and to Mrs. C. Thomas for performing with dispatch the trying task of transcribing the raw data onto punched tape for input to the computer. 


\section{REFERENCES}

1. F. de Hoffmann; The Science and Engineering of Nuclear Power, Vol, 2, Ch. 9, pp. 103-119, Addison Wesley Press, Inc:, (1949).

2. T. F. Ruane, D. J. Anthony, P. Buck, D. A. Gavin, and H. B. Stewart,

"A Measurement of the Effective Delayed Neutron Fraction by the Substitution Method, "Transactions of the American Nuclear Society, Vol. 1, No. 2, Paper 18-8, December 1958.

3. A. F. Henry, Nuclear Science and Engineering, 3, 52 (1958).

4. H. Amster et al, "The Calculation of Thermal Constants Averaged over a Wigner-Wilkins Flux Spectrum: Description of the SOFOCATE Code," WAPD-TM-39, January 1957.

5. H. Bohl, Jr. et al, "MUFT 4-A Fast Neutron Spectrum Code for the IBM-704," WAPD-TM-72, July 1957.*

6. O. J. Marlowe, et al, "WANDA - A One-Dimensional Few-Group Diffusion Equation Code for the IBM-704," WAPD-TM-28, November 1956.

7. G. G. Bilodeau, et al, "PDQ - An IBM-704 Code to Solve the TwoDimensional Few-Group Neutron-Diffusion Equations," WAPD-TM-70, August 1957.

8. H. Bohl, Jro, et al, "A Few-Group one-Dimensional Code for IBM-650," WAPD-TM-3, July 1956.

9. A. F. Henry, "Computation of Parameters Appearing in the Reactor Kinetics Equations, "WAPD-142, December 1955.

10. Lu N. Ussachoff, Geneva Conference Paper No. 656 (1955). 